\title{
TRATAMENTO CIRÚRGICO DO DIVERTÍCULO DE ZENKER
}

\author{
Surgical treatment of Zenker's diverticulum
}

\author{
Guilherme Behrend Silva RIBEIRO, Fernando MIELKE, Bernardo Silveira VOLKWEIS, \\ Carlos Cauduro SCHIRMER, Cléber Dario Pinto KRUEL, Gustavo MORELLATO, \\ Marcelo BINATO, Richard Ricachenevsky GURSKI
}

ABCDDV/605

Ribeiro GBS, Mielke F, Volkweis BS, Schirmer CC, Kruel CDP, Morellato G, Binato M, Gurski RR. Tratamento cirúrgico do divertículo de Zenker. ABCD Arq Bras Cir Dig 2008;21(3):110-3

RESUMO - Racional - O tratamento cirúrgico do divertículo de Zenker inclui na maioria dos casos a cricomiotomia do músculo cricofaríngeo, a qual pode ser associada à diverticulopexia ou diverticulectomia. A escolha destas opções cirúrgicas ainda é controversa. Objetivo - Avaliar os resultados de dois tratamentos cirúrgicos (diverticulopexia ou diverticulectomia, ambos associados à cricomiotomia) em uma série de casos. Métodos - Estudo retrospectivo em período de 10 anos de 26 pacientes submetidos ao tratamento cirúrgico do divertículo de Zenker. Para análise estatística, os pacientes foram divididos em dois grupos: Grupo 1 - diverticulectomia $(n=17)$ e Grupo 2 - diverticulopexia $(n=9)$. Em todos realizou-se miotomia. Foram avaliadas as variáveis: tempo cirúrgico, de internação e de início da alimentação via oral, complicações gerais, ocorrência de fístulas, recidiva dos sintomas e mortalidade. Consideraram-se diferenças significativas quando $P<0.05$. Resultados - A idade média dos pacientes foi de 64 anos. Sintomas pré-operatórios principais: disfagia (91\%) e regurgitação (46\%). Todos foram investigados com estudo radiográfico contrastado de faringe-esôfago-estômago e 58\% dos casos com endoscopia digestiva alta. Não houve diferença significativa entre os Grupos 1 e 2 em relação ao tempo operatório (96 x $99 \mathrm{~min})$, tempo de internação (5,5 x 5 dias), início da alimentação via oral (7,5 x 4 dias), ocorrência de fístulas esôfagocutâneas ( $35 \times 22 \%)$, recidiva da disfagia ( 6 × 11\%), complicações pós-operatórias em geral ( 41 x 33\%) e tempo de seguimento (7,5 x 9 meses). A mortalidade foi nula. Conclusão - O tratamento cirúrgico do divertículo de Zenker é método terapêutico relativamente seguro, com morbidade aceitável e seus resultados independem da opção por ressecção ou pexia do divertículo.

DESCRITORES - Divertículo de Zenker. Cirurgia.

\section{INTRODUÇÃO}

Descrito em 1769 pela primeira vez pelo cirurgião inglês Ludlow19 e posteriormente pelos patologistas alemães Zenker e von Ziemssen ${ }^{30}$, o divertículo faringoesofágico - consagrado como divertículo de Zenker (DZ) -, é na realidade pseudodivertículo, uma vez que consiste apenas das camadas mucosa e submucosa da parede posterior da hipofaringe. Origina-se a partir do músculo constritor inferior da faringe, entre as fibras do constritor faríngeo posterior e o músculo cricofaríngeo (esfíncter esofágico superior).

A incidência desta doença aumenta com a idade, sendo predominantemente encontrada em homens com mais de 60 anos. Inicialmente o divertículo determina abaulamento progressivo na região posterior do esôfago, tornando-se perpendicular a ele. Com a entrada de alimentos e o aumento de pressão, o divertículo vai adquirindo posição vertical até atingir posição paralela ao esôfago. Clinicamente, manifesta-se principalmente por disfagia, regurgitação de alimentos não digeridos, murmúrios no pescoço

Trabalho realizado no Serviço de Cirurgia do Aparelho Digestivo do Hospital de Clínicas de Porto Alegre, Universidade Federal do Rio Grande do Sul, Porto Alegre, RS, Brasil

Correspondência: Richard. R. Gurski, e-mail: richard@ portoweb.com.br após alimentação, halitose e emagrecimento. Sintomas compressivos e complicações respiratórias ocasionais, como broncoaspiração e pneumonia, podem ocorrer. Como o principal sintoma é disfagia e o acometimento é predominante em pacientes com mais de 60 anos, outras afecções esofágicas devem ser investigadas no diagnóstico diferencial: neoplasias, hérnia hiatal, megaesôfago, estenose esofágica péptica ou química e doença do refluxo gastroesofágico. $\mathrm{O}$ exame contrastado da faringe-esôfagoestômago é o principal método diagnóstico. A endoscopia digestiva alta e a manometria auxiliam no diagnóstico diferencial, porém esses exames devem ser realizados com cautela para evitar perfuração do esôfago ${ }^{10}$.

Dois fatores parecem ser essenciais na sua patogênese: o aumento da pressão na hipofaringe durante a deglutição e a diminuição da resistência da sua parede posterior ao nível do triângulo de Killian ${ }^{10}$. Controvérsias persistem sobre a importância de cada um destes fatores na formação do DZ. Cook et al. ${ }^{5}$, através de estudo videofluoroscópico e manométrico simultâneos, avaliaram a fisiopatologia da obstrução ao fluxo dos alimentos durante a deglutição. Os autores concluíram que o DZ é desordem relacionada à diminuição da abertura do esfíncter esofágico superior (EES), a qual não seria causada por incoordenação motora 
ou relaxamento inadequado dele, mas pela sua abertura incompleta. Assim, ocorreria aumento da pressão hipofaríngea consequentemente à inadequada passagem de alimentos. Entretanto, outros autores ainda sustentam que ocorre relaxamento incompleto do EES, resultante de fibrose localizada ${ }^{4,29}$.

O manejo do DZ é cirúrgico e busca o alívio dos sintomas, entre eles a disfagia, a regurgitação e a prevenção da pneumonia de aspiração. Várias abordagens foram desenvolvidas, incluindo diverticulectomia e diverticulopexia transcervical com ou sem cricomiotomia e as técnicas minimamente invasivas (tratamento endoscópico com stapler ou laser) ${ }^{9}$. Estas últimas possuem os benefícios inerentes aos procedimentos minimamente invasivos, mas têm maior taxa de recidiva e risco de perfuração esofágica ${ }^{6,12,22}$, além de possuírem limitações técnicas e menor disponibilidade em nosso meio. Quanto à cricomiotomia associada, apesar de haver alguma discussão quanto à necessidade de ser realizada em todos os pacientes, provavelmente acarreta melhores resultados a longo prazo, possivelmente por tratar uma das causas do $\mathrm{DZ}^{3,7,12,25,29}$.

Em estudo recente, o critério para realização de diverticulectomia foi diâmetro do divertículo superior a 3 $\mathrm{cm}$, e diverticulopexia para divertículos com menos de 3 $\mathrm{cm}^{12}$. Nesta casuística, iniciada em 1994, não houve avaliação transoperatória objetiva (e.g., uso de régua), porém o tamanho dos divertículos foi subjetivamente avaliado e, então, optou-se pela diverticulectomia nos maiores e diverticulopexia nos menores.

Tendo-se em vista as diversas abordagens cirúrgicas factíveis e a escassa literatura nacional a respeito da experiência no tratamento dessa doença pouco prevalente, este estudo tem por objetivo avaliar os resultados do tratamento cirúrgico do DZ em uma série de casos.

\section{MÉTODOS}

Foi conduzido estudo retrospectivo de pacientes com DZ submetidos a tratamento cirúrgico pelo Grupo de Cirurgia do Esôfago-Estômago-Intestino Delgado do Hospital de Clínicas de Porto Alegre, no período de outubro de 1994 a abril de 2006. Todos realizaram cricomiotomia, a qual era associada à diverticulectomia ou diverticulopexia. Os dados clínicos e cirúrgicos foram aferidos por revisão de prontuários posteriormente à aprovação do Grupo de Pesquisa e Pós-Graduação do Hospital de Clínicas de Porto Alegre.

Todos pacientes foram submetidos a tratamento cirúrgico através de incisão cervical esquerda na borda medial do músculo esternocleidomastoideo. A decisão entre ressecção ou pexia do divertículo foi definida no transoperatório. Todos realizaram cricomiotomia.

Os pacientes foram avaliados quanto aos aspectos clínicos, radiológicos e endoscópicos pré-operatórios, quais sejam: sexo, idade, disfagia e seu tempo de evolução, regurgitação, emagrecimento e outros sintomas quaisquer (pneumonia aspirativa, halitose, disfonia, pirose), registro de estudo contrastado e/ou endoscopia digestiva alta e as alterações associadas ao DZ encontradas nesses exames.

Os aspectos cirúrgicos, clínicos, radiológicos e en- doscópicos pós-operatórios aferidos na revisão de prontuários foram: tratamento cirúrgico, tempo operatório, tempo de internação, tempo para o início da alimentação via oral, complicações, óbito, recidiva de disfagia, recidiva de regurgitação, tempo de acompanhamento, registro de estudo contrastado e/ou endoscopia digestiva alta e as alterações encontradas nesses exames.

Para análise, os pacientes foram divididos em dois grupos, conforme o tratamento cirúrgico executado. Quando submetidos a diverticulectomia e cricomiotomia, Grupo $1(\mathrm{n}=17)$; quando à diverticulopexia e cricomiotomia, Grupo $2(\mathrm{n}=9)$.

Para a análise estatística das variáveis contínuas foram utilizados o teste $\mathrm{t}$ de Student para amostras independentes e simétricas ou Mann-Whitney se assimétricas, para as variáveis categóricas foram utilizados o teste de quiquadrado ou teste exato de Fisher. Foi considerado um nível de significância de 5\%.

\section{RESULTADOS}

Foram estudados 26 pacientes, dos quais 17 submetidos à cricomiotomia e diverticulectomia (Grupo 1), e nove à cricomiotomia e diverticulopexia (Grupo 2).

Os aspectos pré-operatórios de ambos os grupos foram analisados em conjunto, exceto a idade média e a distribuição por sexos. Em análise comparativa quanto à idade média (70 e 59 anos) e à distribuição de sexo ( $53 \%$ e $56 \%$ homens), não houve diferença significativa $(P>0.05)$ entre os Grupos 1 e 2, respectivamente. Avaliando-os globalmente $(\mathrm{n}=26)$, disfagia esteve presente em 25 (96\%) pacientes, regurgitação em 12 (46\%), emagrecimento em 4 (15\%), vômitos em 3 (11\%), halitose em $2(8 \%)$, pirose em $2(8 \%)$, pneumonia aspirativa em 2 (8\%), disfonia em 1 (4\%) e odinofagia em 1 (4\%). Durante a investigação diagnóstica, o estudo contrastado foi realizado nos $26(100 \%)$ pacientes e a endoscopia digestiva alta em 15 (58\%). Além da identificação do DZ na radiografia em $100 \%$ dos pacientes, foram encontradas alterações associadas em 5 pacientes: hérnia hiatal em 2, estenose do esôfago proximal em 1, redução do calibre do esôfago distal ao nível da cárdia com dilatação a montante em 1 e divertículo menor associado ao DZ localizado na parede lateral direita em 1 paciente. Na endoscopia digestiva alta foram encontradas alterações associadas ao DZ em 7 pacientes, quais sejam: 2 com hérnia hiatal somente, 2 com gastrite, 1 com hérnia hiatal e Barret, 1 com gastrite e esofagite, e 1 com hérnia hiatal, gastrite e duodenite associadas.

$\mathrm{Na}$ análise dos grupos, não houve diferença significativa no tempo operatório médio (96 e $99 \mathrm{~min}$ ), no tempo de internação (5,5 e 5 dias) e no início da alimentação via oral (7,5 e 4 dias) com $P>0.05$ (Tabela 1).

TABELA 1 - Períodos de tempo estudado nos pacientes

\begin{tabular}{llllll}
\hline Tempo & n & G 1 & n & G 2 & $\boldsymbol{P}$ \\
\hline Operatório (min)* & 16 & $95,6 \pm 21,8$ & 8 & $99,4 \pm 22,6$ & 0,7 \\
Internação (dias) & 17 & $5,5(3-12)$ & 9 & $5(4,5-14)$ & 0,94 \\
Início da alimentação VO (dias) & 17 & $7,5(3-10,5)$ & 9 & $4(3-16)$ & 0,89 \\
Acompanhamento (meses) & 16 & $7,5(2,49-23,7)$ & 9 & $9,1(3,8-19,7)$ & 0,86 \\
\hline
\end{tabular}

* Média + DP. Demais apresentados com mediana (percentis 25 e 75\%). 
Quanto às complicações pós-operatórias (Gráfico 1), no Grupo 1 houve formação de fístula esofagocutânea em 6 (35\%) pacientes, infecção da ferida operatória em $1(6 \%)$ e qualquer complicação em 7 (41\%). No Grupo 2, houve formação de fístula esôfago-cutânea em 2 (22\%) pacientes, infecção da ferida operatória em $1(11 \%)$ e qualquer complicação em 3 (33\%). No Grupo 1 ocorreu um infarto agudo do miocárdio no transoperatório e o paciente foi encaminhado ao unidade de terapia intensiva, com boa evolução. Nenhuma dessas complicações diferiu significativamente entre os grupos.

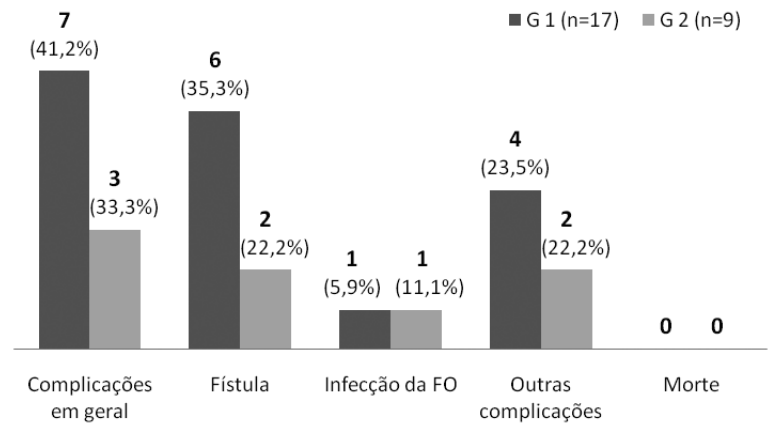

Dados apresentados com n (\%)

Nenhuma variável foi significativamente diferente entre os grupos $(P>0,05)$.

GRÁFICO 1 - Complicações pós-operatórias

Quanto à recidiva dos sintomas (Gráfico 2), houve duas (8\%) recidivas de disfagia, uma em cada grupo, ocasionando sintomas diários. Os resultados foram satisfatórios em 24 (92\%) pacientes.

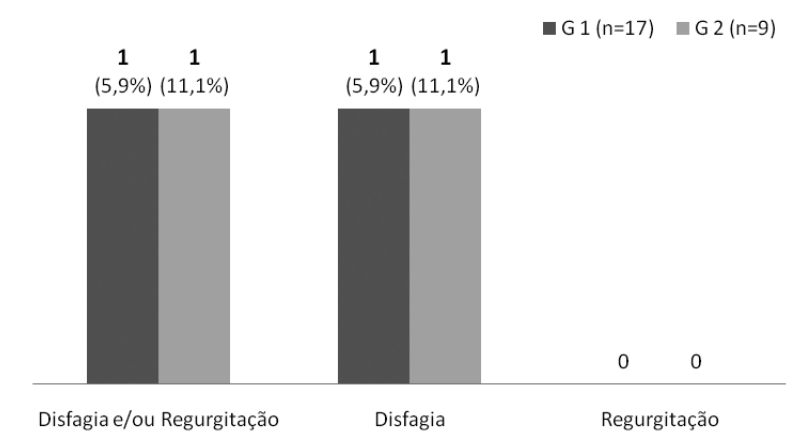

Dados apresentados com n (\%)

Nenhuma variável foi significativamente diferente entre os grupos $(P>0,05)$.

GRÁFICO 2 - Recidiva dos sintomas

As medianas dos tempos de seguimento também não diferiram significativamente entre o Grupo 1 (7,5 meses) e o Grupo 2 ( 9 meses) $(P>0.05)$.

\section{DISCUSSÃO}

O tratamento do DZ é fundamentalmente cirúrgico, porém discute-se qual seria o manejo mais adequado. Várias abordagens cirúrgicas foram propostas: cricomiotomia isolada, cricomiotomia associada à ressecção ou pexia do divertículo, diverticulectomia isolada, diverticulopexia isolada, ressecção endoscópica com grampeador, laser ou coagulação ${ }^{1}$. Tendo-se em vista que o DZ é doença rara, mais comumente encontrada nos países nórdicos da Europa $^{28}$, o desenho de ensaios clínicos randomizados, prospectivos e cegos torna-se tarefa árdua. Observando a literatura mundial, nota-se razoável número de publicações avaliando os resultados das diversas técnicas cirúrgicas, porém a maioria é de baixa qualidade metodológica ${ }^{2,3,4,12,15,17,18}$. Apesar das técnicas endoscópicas estarem cada vez mais difundidas, especialmente na Europa (por exemplo, no Reino Unido 83\% dos cirurgiões de cabeça e pescoço preferem o método ${ }^{27}$, na América Latina há poucos relatos demonstrando seu uso no tratamento do $\mathrm{DZ}^{14,23,24}$. Em verdade, mesmo a publicação dos tratamentos convencionais é relativamente escassa ${ }^{13,20,21}$.

Neste estudo realizou-se análise comparativa dos resultados intra e pós-operatoriamente dos dois tratamentos realizados. Observou-se que os dois grupos apresentaram resultados estatisticamente semelhantes quanto aos seguintes aspectos: tempo cirúrgico, de hospitalização e de início da alimentação via oral, complicações gerais, ocorrência de fistulas, recidiva dos sintomas e mortalidade ( $P>0.05$ em todos os desfechos). Pode-se afirmar, por conseguinte, que as duas técnicas podem ser utilizadas, apresentando evolução, complicações pós-operatórias e recidiva dos sintomas em taxas satisfatórias, comparáveis com os estudos de maior porte ${ }^{3,12,17,18}$.

Com relação a rotina de sempre realizar cricomiotomia, há, de fato, evidências contraditórias em relação à superioridade da ressecção ou pexia associados à cricomiotomia, quanto aos resultados pós-operatórios e taxa de recidivas ${ }^{3,12,18}$. Idealmente, a decisão de miotomia ou não deveria ser embasada em exame pré-operatório capaz de identificar com boa acurácia a disfunção do músculo cricofaríngeo ${ }^{3}$. No entanto, inexiste tal exame. Logo, é impossível avaliar essa disfunção e identificar no pré-operatório aqueles pacientes que deveriam realizar ou não a miotomia. Além disso, há evidências que suportam aumento da formação de fístulas e de outras morbidades naqueles pacientes que não foram submetidos à miotomia ${ }^{8,12,16}$. Desse modo, preconiza-se sua realização rotineira.

Por fim, a utilização de técnicas endoscópicas está cada vez mais difundida, apresentando benefícios como menor tempo cirúrgico, menor tempo de hospitalização, início da alimentação via oral mais rápido e menor tempo de recuperação $0^{2,6,11,26}$. Porém, em estudo restrospectivo de Gutschow et al. ${ }^{12}$, que avaliou os resultados de seis diferentes técnicas (cricomiotomia isolada, cricomiotomia e diverticulectomia, cricomiotomia e diverticulopexia, diverticulectomia isolada, diverticulopexia isolada, ressecção endoscópica com grampeador e ressecção endoscópica com laser), as técnicas transcervicais resultaram em melhor alívio sintomático, principalmente em pacientes com divertículos pequenos. 


\section{CONCLUSÃO}

A decisão de que tipo de tratamento melhor se aplica a cada paciente com DZ deverá considerar principalmente a experiência local, o risco cirúrgico do paciente e o ta- manho do divertículo, preferindo-se a diverticulectomia à diverticulopexia nos pacientes de baixo risco cirúrgico e/ou com divertículos maiores de $3 \mathrm{~cm}$ realizando-se de rotina a cricomiotomia.

Ribeiro GBS, Mielke F, Volkweis BS, Schirmer CC, Kruel CDP, Morellato G, Binato M, Gurski RR. Surgical treatment of Zenker's diverticulum. ABCD Arq Bras Cir Dig 2008;21(3):110-3

ABSTRACT - Background - Surgical treatment of Zenker's Diverticulum comprise in the majority of cases cricopharyngeal miotomy, which may be associated with diverticulopexy or diverticulectomy. The election of these surgical options remains controversial. Aim - To evaluate the results of two surgical treatments (diverticulopexy or diverticulectomy, both associated with miotomy) in a case series. Methods - Retrospective study comprising a 10-year period of 26 patients submitted to surgical treatment of Zenker's Diverticulum. For statistical analysis, patients were divided in two groups: Group 1 - diverticulectomy $(\mathrm{n}=17)$, and Group 2 - diverticulopexy $(\mathrm{n}=9)$. All were submitted also to a cricomiotomy. The following variables were evaluated: operative time, in-hospital stay, time to initiate oral feeding, general complications, fistula occurrence, symptom recurrence and mortality. Significant differences considered when $P<0.05$. Results - Mean age was 64 years. Main pre-operative symptoms were: dysphagia $(91 \%)$ and regurgitation (46\%). All patients were diagnosed with pre-operative barium esophagram and $58 \%$ ( $\mathrm{n}=15)$ did upper gastrointestinal endoscopy. There wasn't significant difference related to mean operative time (96 x $99 \mathrm{~min})$, in-hospital stay (5,5 x 5 days), time to initiate oral feeding (7,5 4 days), occurrence of esophagocutaneous fistula $(35 \times 22 \%)$, dysphagia recurrence $(6 \times 11 \%)$, general post-operative complications (41 x 33\%) and follow up period ( 7,5 x 9 months). The mortality was null. Conclusion - Surgical treatment of Zenker's Diverticulum is a relatively safe therapeutic method, with acceptable morbidity and the results are comparable between diverticulopexy and diverticulectomy.

HEADINGS - Zenker diverticulum. Surgery.

\section{REFERÊNCIAS}

1. Aly A, Devitt PG, Jamieson GG. Evolution of surgical treatment for pharyngeal pouch. Br J Surg. 2004 Jun;91(6):657-64

2. Chang CY, Payyapilli RJ, Scher RL. Endoscopic staple diverticulostomy for Zenker's diverticulum: review of literature and experience in 159 consecutive cases. Laryngoscope. 2003 Jun;113(6):957-65.

3. Colombo-Benkmann M, Unruh V, Krieglstein C, Senninger N. Cricopharyngeal myotomy in the treatment of Zenker's diverticulum. J Am Coll Surg. 2003 Mar;196(3):370-7.

4. Cook IJ, Blumbergs P, Cash K, Jamieson GG, Shearman DJ. Structural abnormalities of the cricopharyngeus muscle in patients with pharyngeal (Zenker's) diverticulum. J Gastroenterol Hepatol. 1992 Nov-Dec;7(6):556-62.

5. Cook IJ, Gabb M, Panagopoulos V, Jamieson GG, Dodds WJ, Dent J, Shearman DJ. Pharyngeal (Zenker's) diverticulum is a disorder of upper esophageal sphincter opening. Gastroenterology. 1992 Oct;103(4):1229-35.

6. Cook RD, Huang PC, Richstmeier WJ, Scher RL. Endoscopic staple-assisted esophagodiverticulostomy: an excellent treatment of choice for Zenker's diverticulum. Laryngoscope. 2000 Dec;110(12):2020-5.

7. DeMeester T, Bremner CG. Selective cricopharyngeal myotomy for Zenker's diverticulum. J Am Coll Surg. 2003 Mar;196(3):451-2.

8. Duranceau A, Rheault MJ, Jamieson GG. Physiologic response to cricopharyngeal myotomy and diverticulum suspension. Surgery. 1983 Oct;94(4):655-62.

9. Ferguson MK. Evolution of therapy for pharyngoesophageal (Zenker's) diverticulum. Ann Thorac Surg. 1991 May;51(5):848-52.

10. Ferreira LE, Simmons DT, Baron TH. Zenker's diverticula: pathophysiology, clinical presentation, and flexible endoscopic management. Dis Esophagus. 2008;21(1):1-8.

11. Fremling C, Raivio M, Karppinen I. Endoscopic discision of Zenker's diverticulum. Ann Chir Gynaecol. 1995;84(2):169-72.

12. Gutschow CA, Hamoir M, Rombaux P, Otte JB, Goncette L, Collard JM. Management of pharyngoesophageal (Zenker's) diverticulum: which technique? Ann Thorac Surg. 2002 Nov;74(5):1677-82.

13. Hajar N, Malafaia O, Strobel R, Cantarelli A, Coelho JCU. Divertículo de Zenker: estudo retrospectivo de casos. Rev Méd Paraná 2000; 58:17-23.

14. Ivano FH, Nicollelli GM, Silveira F, Contin CM, Batista M, Silva CM, Sasaki CA, Miyake RT, Kay AK, Hasegawa C. Tratamento clínco de perfuração esofágica após tratamento endoscópico de divertículo de Zenker: relato de caso. Gastroenterol Endosc Dig 2002; 21:221-223.

15. Jougon J, Le Taillandier-de-Gabory L, Raux F, Delcambre F, Mac Bride T, Velly JF. Plea in favour of external cervicotomy approach of Zenker's diverticulum: 73 cases reported. Ann Chir. 2003 Apr;128(3):167-72.

16. Konowitz PM, Biller HF. Diverticulopexy and cricopharyngeal myotomy: treatment for the high-risk patient with a pharyngoesophageal (Zenker's) diverticulum. Otolaryngol Head Neck Surg. 1989 Feb;100(2):146-53.
17. Leporrier J, Salamé E, Gignoux M, Ségol P. Zenker's diverticulum: diverticulopexy versus diverticulectomy. Ann Chir. $2001 \mathrm{Feb} ; 126(1): 42-5$.

18. Lerut T, van Raemdonck D, Guelinckx P, Dom R, Geboes K. Zenker's diverticulum: is a myotomy of the cricopharyngeus useful? How long should it be? Hepatogastroenterology. 1992 Apr;39(2):127-31.

19. Ludlow A. A case of obstructed deglutition from a preternatural bag formed in the pharynx. Johnson W, Caldwell T, ed. In: Medical observations and inquiries by a society of physicians in London. London, Vol. 3, 2nd ed. 1769:85-101.

20. Muraro CPM, Aquino JLB, Silva MRM, Lintz JE. Divertículo de Zenker: análise de 11 pacientes. Rev Ciênc Méd. 1997;6:95-8.

21. Okano N, Vargas EC, Moriya T, Carneiro JJ, Elias Jr AM. Divertículo do esôfago análise de 24 pacientes portadores do divertículo de Zenker. Acta Cir Bras 2000; 15(supl.2):60-2.

22. Philippsen LP, Weisberger EC, Whiteman TS, Schmidt JL. Endoscopic stapled diverticulotomy: treatment of choice for Zenker's diverticulum. Laryngoscope. 2000 Aug;110(8):1283-6.

23. Sakai P. Endoscopic treatment of Zenker's diverticulum. Gastrointest Endosc. 2007 Jun;65(7):1054-5.

24. Santos RR, Machado M, Kupski C. Tratamento endoscópico do divertículo de Zenker: relato de dois casos. Rev AMRIGS 1995; 39: 331-4.

25. Shaw DW, Cook IJ, Jamieson GG, Gabb M, Simula ME, Dent J. Influence of surgery on deglutitive upper oesophageal sphincter mechanics in Zenker's diverticulum. Gut. 1996 Jun;38(6):806-11.

26. Siddiq MA, Patel PJ. Pharyngeal pouch surgery: a five year review. Rev Laryngol Otol Rhinol (Bord). 2000;121(1):37-40.

27. Siddiq MA, Sood S. Current management in pharyngeal pouch surgery by UK otorhinolaryngologists. Ann R Coll Surg Engl. 2004 Jul;86(4):247-52.

28. van Overbeek JJ. Pathogenesis and methods of treatment of Zenker's diverticulum. Ann Otol Rhinol Laryngol. 2003 Jul;112(7):583-93.

29. Zaninotto G, Costantini M, Boccù C, Anselmino M, Parenti A, Guidolin D, Ancona E. Functional and morphological study of the cricopharyngeal muscle in patients with Zenker's diverticulum. Br J Surg. 1996 Sep;83(9):1263-7.

30. Zenker FA, von Ziemssen H. Krankheiten des Oesophagus. In: von Ziemssen H, ed. Handbuch der speciellen Pathologie und Therapie, vol. 7 (suppl.). Leipzig: FC Vogel, 1877:1-87.
Fonte de financiamento: não há Conflito de interesse: não há Recebido para publicação: 03/02/2008 Aceito para publicação: 28/04/2008 